\title{
Fausto Goria
}

\section{Zur ehelichen Gütergemeinschaft im philosophischen Denken der Griechen von Xenophon bis Ioannes Stobaios}

1. Die Frage, ob und gegebenenfalls in welchem Umfang die Ehegemeinschaft im oströmischen Reich Auswirkungen auf die vermögensrechtlichen Beziehungen der Ehepartner hatte, kann unter verschiedenen Gesichtspunkten behandelt werden. Nicht außer acht gelassen werden sollten dabei meines Erachtens die damals verbreiteten philosophischen Vorstellungen über das Wesen der Ehe, ihre Ziele und die beste Art und Weise, sie zu verwirklichen; bei Erörterung dieser Thematik konnte unter Umständen auch der ökonomische Aspekt des Familienlebens zur Sprache kommen.

Meines Wissens wurde eine solche Untersuchung bislang nicht in Angriff genommen; sicherlich bedarf sie einer Vorstudie über die diesbezüglichen Äußerungen von Autoren aus den Jahrhunderten vor Konstantinos dem Großen, weil ihre Schriften auch noch in der Folgezeit im Originaltext gelesen wurden und darüber hinaus in Auszügen Eingang in doxographische Kompilationen fanden, von denen jene des Ioannes aus Stoboi (einer Stadt in Mazedonien) wohl vom Beginn des 5. Jahrhunderts n. Chr. fast vollständig erhalten blieb.

Die vorliegende Arbeit versteht sich als Beitrag zu einer solchen Vorstudie; es sollen hier diejenigen Schriften untersucht werden, in denen mehr oder weniger deutlich von ehelicher Gütergemeinschaft die Rede ist ${ }^{1}$, und dabei soll möglichst jeweils ermittelt werden, in welchem Sinne (insbesondere in bezug auf die juristische Fragestellung) der Begriff verwendet wurde und in welcher Beziehung er zum allgemeinen Ehekonzept stand. Zu diesem Zweck wurden die Kapitel 22 und 23 des 4 . Buches des Anthologion des Ioannes Stobaios herangezogen, welche der Ehe und den $\gamma \alpha \mu$ ik

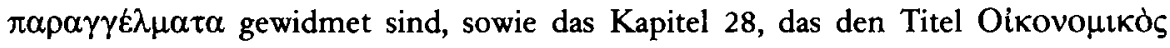

${ }^{1}$ Die redaktionell bedingte Eile, mit der der vorliegende Beitrag abgefaßt werden mußte, erlaubte weder eine Ermittlung noch eine Diskussion der gesamten diesbezüglichen Literatur. Zitiert werden daher nur jene Werke, die zur Ergānzung und Vertiefung unserer Ausführungen unentbehrlich erschienen. Eine erste - auch bibliographische - Orientierung über die hier behandelte Problematik bietet: Für und wider die Ehe. Antike Stimmen zu einer offenen Frage zusammengestellt und übersetzt von Konrad Gaiser (München 1974) 59-88 (im folgenden Gaiser, Ehe). Die für die griechischen Schriftsteller und deren Werke (mit Ausnahme von Plutarchs Moralia) benützten Abkürzungen richten sich nach Henry George Liddell-Robert Scott, A GreekEnglish Lexicon ... with a Supplement 1968 (Oxford 1976). 
trägt ${ }^{2}$. Von den genannten Stellen wurden jedoch zum einen nur solche Passagen berücksichtigt, die auf Philosophen, nicht aber auf Tragiker und Komiker zurückgehen; zum anderen schien es unangemessen, sich auf das im Anthologion zusammengestellte Material zu beschränken, weswegen auch andere einschlägige, im weitesten Sinne philosophische Zeugnisse in die Untersuchung mit einbezogen wurden. Zwar wurde versucht, nichts Wesentliches außer acht zu lassen, eine absolute Vollständigkeit wurde jedoch nicht angestrebt; vielmehr mußte mehrfach eine Auswahl vorgenommen werden, welche notwendigerweise nicht frei von Willkür ist: So erschien es zweckmäßig, Autoren aus der Zeit vor dem 4.Jahrhundert v. Chr. (welches - zumindest von der literarischen Produktion her gesehen - das goldene Jahrhundert der griechischen Philosophie ist) unberücksichtigt zu lassen. Aber auch Platon, dessen Ansichten in bezug auf Ehe und Familie nur im weiteren Rahmen seines Entwurfs einer Idealgesellschaft begriffen werden können, blieb außer Betracht. Andererseits wurde, wenngleich erst die Kompilation des Ioannes Stobaios vom Anfang des 5.Jahrhunderts n. Chr. den Endpunkt der Quellenuntersuchung bildet, auf eine Darstellung der Auffassungen christlicher Schriftsteller verzichtet, um den Umfang der vorliegenden Arbeit nicht allzusehr auszudehnen, aber auch, um dem Umstand Rechnung zu tragen, daß sich Stobaios selbst auf die Wiedergabe von Zeugnissen der heidnischen Kultur beschränkte. Im übrigen schenkte die Patristik der Ehe zwar natürlich große Aufmerksamkeit, nicht aber deren ökonomischen Aspekten, weswegen die Frage der vermögensrechtlichen Beziehungen zwischen Ehegatten im patristischen Schrifttum eine vergleichsweise bescheidene Rolle spielt.

2. Der erste für unser Thema einschlägige Autor ist Xenophon. In seinem „Oikonomikos“ (der vielleicht zwischen 390 und 371 auf Xenophons Landgut bei der in Elis gelegenen Ortschaft Skillus verfaßt wurde) werden keine Ratschläge erteilt, sondern Erfahrungen des Ehealltags beschrieben, und zwar diejenigen eines Athener Bürgers namens Ischomachos, der - als teilweise idealisiertes Spiegelbild des Autors - nach

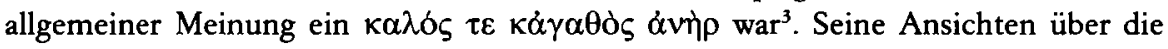
Ehe erklärt er, indem er die Belehrungen an seine Frau referiert. Zweck der Ehe sei danach die Entwicklung einer bestmöglichen Gemeinschaft in bezug auf das Hauswesen und die Kinder; können doch diese besonders insofern ein gemeinsames Gut beider Eltern darstellen, als sie sie im Alter unterstützen werden; nun aber sei auch in der Zwischenzeit das Haus, in dem die Ehegatten leben, beider gemeinsames Gut. So habe er seiner Frau erklārt, seine ganzen eigenen Vermögensgegenstānde der Gemeinschaft zuzurechnen, während sie ihrerseits in die Gemeinschaft all das eingebracht habe, was sie erhalten habe. Grundsatz einer solchen Vereinigung sei es, daß nicht etwa derjenige, der eine größere Zahl an Vermögensgegenständen eingebracht habe,

2 Ioannis Stobaei Anthologium, rec. C. Wachsmuth et O. Hense, vol.IV, Anthologii libri quarti partem priorem ab O. Hense editam continens (Berolini 1909) 495-599 (über die Ehe und die $\gamma \alpha \mu \iota \kappa \dot{\alpha} \pi \alpha \rho \alpha \gamma \gamma \hat{\varepsilon} \lambda \mu \alpha \tau \alpha)$; vol.V, Anthologii libri quarti partem alteram ab O. Hense editam continens (Berolini 1912) 677-701 (Oikovouıкós).

${ }^{3}$ X. Oec. VI,12. 
sondern derjenige, der der bessere Gesellschafter sei, den wertvolleren Beitrag geleistet habe ${ }^{4}$.

Um die vorhandenen Güter nun möglichst zweckmäßig zu bewahren und (selbstverständlich unter Wahrung von Recht und Billigkeit), soweit möglich, zu vermehren, sei ein Zusammenwirken beider Ehegatten erforderlich, wobei sich die Aufgabenteilung nach den geschlechtsspezifischen Fähigkeiten richten solle ${ }^{5}$ : Der Ehemann werde demnach die Feldarbeiten und überhaupt die außerhäuslichen Tätigkeiten verrichten (welche in der Regel auch die "produktiven“ seien) ${ }^{6}$; die Ehefrau dagegen werde sich um die Aufbewahrung der Vorräte und generell um den Haushalt kümmern ${ }^{7}$, für Verpflegung und Kleidung sorgen (also für die „Verarbeitung “), die Kleinkinder aufziehen, die Haussklaven ${ }^{8}$ anweisen, überwachen, vielleicht auch unterrichten, die Kranken pflegen und die Gegenstände des täglichen Verbrauchs austeilen und unter ihrer Kontrolle halten. Da ihr die Güter selbst gehörten, werde es ihr schließlich größere Genugtuung bereiten, ihnen Sorgfalt angedeihen zu lassen, als sie zu vernachlässigen ${ }^{10}$. Etwas später wird die Stellung von Ehegatten mit derjenigen von Teil-

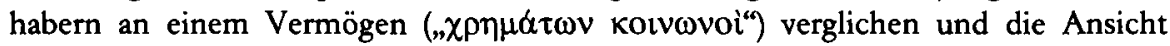
vertreten, daß die Ehegatten auch an den eigenen Körpern wechselseitigen Anteil hātten ${ }^{11}$.

Allen diesen Erörterungen ist natürlich eine spezifisch juristische Sichtweise fremd. Höchstwahrscheinlich ist nämlich die feierliche Aussage „'E $\gamma \dot{\omega} \tau \varepsilon \ldots \delta \sigma \alpha \mu o \iota$ ह̌ $\sigma \tau \iota v$

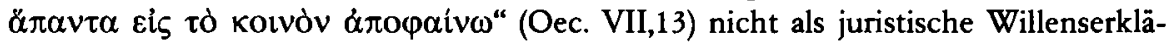
rung zur Begründung wechselseitigen Miteigentums an allen Vermögensgegenständen der Ehegatten zu werten (dies hätte auch keineswegs athenischem Brauch entsprochen), ganz abgesehen davon, daß diese Aussage, selbst wenn dies beabsichtigt gewesen wäre, zur Erreichung des juristischen $Z$ weckes kaum ausreichend gewesen wäre. Im übrigen befaßt sich Xenophon mit der Erhaltung und Vermehrung des Vermögens und geht daher nicht auf die Veräußerung einzelner Gegenstände oder Vermögensteile ein, deren Erörterung ihm vielleicht Anlaß zu Präzisierungen geboten hätte. Trotzdem glaube ich nicht, daß die zitierten Worte lediglich dazu dienen, die übliche Gütergemeinschaft von Personen, die unter ein und demselben Dach wohnen, eindringlich zu umschreiben; ich meine vielmehr, daß diese Worte einerseits das Versprechen beinhalten, dem Haushalt sämtliche Erträgnisse des Vermögens zukommen zu lassen und sie also der Ehefrau zur Verfügung zu stellen, andererseits dem Wunsch Ausdruck verleihen, die Gattin an der Vermögensverwaltung zu beteiligen.

Gerade auf diesen letzten Punkt legt Ischomachos besonderen Nachdruck, indem er minuziös die von den Ehegatten jeweils zu erfüllenden Aufgaben beschreibt und dabei deren Verschiedenheit mit dem Hinweis auf die verschiedenen natürlichen

4 Ibid., VII,11-13. Eine deutsche Ưbersetzung bietet Gaiser, Ehe 24.

5 Ibid., VI,15ff.; $22 \mathrm{ff}$.

6 Vgl. auch ibid., Kap. XIff.

7 Vgl. auch ibid., VIII,10 ff.

8 Vgl, auch ibid., X,10.

9 Ibid., VII,36; vgl. auch III,15.

10 Ibid., IX,19.

${ }^{11}$ Ibid., X,3-5. 
Fähigkeiten der beiden Geschlechter rechtfertigt. Daraus kann man wohl folgern, daß die am Beginn der Abhandlung genannte Gütergemeinschaft im folgenden Sinne zu verstehen ist: Die Vermögen der beiden Gatten sollen fortan eine einzige ungeteilte Masse bilden, damit bei ihrer Verwaltung - zum größtmöglichen wirtschaftlichen Nutzen und unabhängig von den jeweiligen juristischen Eigentumsverhältnissen eine geschlechtsspezifische Aufgabenteilung vorgenommen werden kann. Wenn das Familienvermögen in dieser Weise als eine Einheit angesehen wird, welche beiden Partnern unterschiedslos gehört, läßt sich auch die Forderung leichter rechtfertigen, die Ehefrau müsse all die zahlreichen Verpflichtungen übernehmen, wie sie ein Haushalt wie derjenige des Ischomachos nun einmal auferlege. Andererseits liege der Wert der ehelichen Gemeinschaft - so Ischomachos vielleicht in versteckter Polemik gegen die Ansprüche reich ausgestatteter Ehefrauen - gerade in der Tätigkeit jedes Partners und nicht etwa in der Menge der Güter, die man in die gemeinsame Vermögensmasse eingebracht habe.

Man könnte nun einwenden, daß der Frau somit ein Wirkungskreis zugewiesen wurde, der jedenfalls streng auf den Bereich des Hauses beschränkt sei, wohingegen das gesamte übrige Vermögen der Kontrolle des Mannes unterstehe; in der Sicht Xenophons ist aber der olkoৎ der - auch wirtschaftliche - Mittelpunkt des Familienlebens, der Ort, zu dem die Einnahmen und aus dem die Ausgaben fließen. Dementsprechend konnte Sokrates in dem nämlichen Dialog - unter Vorwegnahme des später von Ischomachos skizzierten Bildes - behaupten, daß die Einnahmen eines Haushaltes durch die Tätigkeit des Mannes erwirtschaftet würden, daß die Ausgaben aber in der Regel durch die Frau verwaltet würden; so sei der Beitrag einer guten Ehefrau zur häuslichen Gemeinschaft demjenigen des Ehemannes gleichwertig ${ }^{12}$.

Xenophons Eheideal beruht hier, bar jeglicher Romantik, auf einer rein wirtschaftlichen Grundlage; die soeben umrissene „Gütergemeinschaft" erweist sich indes als ein interessanter Versuch, die Ehe als eine einigermaßen „paritätische“ Gemeinschaft darzustellen (abgesehen von der Vorrangstellung, die der Ehemann - in Gestalt des Ischomachos - als planender Leiter bezüglich der Aufgaben des Paares einnimmt), als eine Gemeinschaft, in der die Gatten gleiche Bedeutung, aber unterschiedliche Funktionen haben, welche mehr oder weniger unmittelbar das gesamte gemeinsame Vermögen betreffen ${ }^{13}$.

3. Es kann hier begreiflicherweise nicht untersucht werden, ob und gegebenenfalls wie Xenophons Modell praktische Relevanz für griechische Poleis des 4.Jahrhunderts und anderswo haben konnte. In theoretischer Hinsicht aber bekräftigte Aristoteles in der

12 Ibid., III,15.

13 Zum Ehemann als Lehrer der Frau (eine auch in X. Smp. II,9 vertretene Ansicht) vgl. auch X. Oec. III,10-14; die diesbezügliche Position des Philodemos von Gadara analysiert Renato Laurenti, Filodemo e il pensiero economico degli epicurei (Testi e documenti per lo studio dell'antichità, XXXIX, Milano 1973) 40-46. Der neue Aspekt des Modells Xenophons besteht nicht darin, daß der Mann seine Frau unterweist, und schon gar nicht in der skizzierten Aufgabenteilung unter den Eheleuten, sondern darin, daß beiden Aufgabenbereichen gleiche Bedeutung beigemessen wird und daß sie als zweckmäßig im Hinblick auf eine gemeinsame Vermögensmasse erachtet werden. 
zweiten Hälfte des Jahrhunderts die Notwendigkeit einer Aufgabenteilung und gegenseitigen Ergänzung der Ehegatten, allerdings ohne - zumindest in den erhaltenen Werken - eine systematische und vertiefte Behandlung der Problematik des Ehelebens zu bieten. Die in diesem Zusammenhang vielleicht wichtigste Stelle findet sich in der Nikomachischen Ethik: Hier wird die Beziehung zwischen Mann und Frau im Bereich der Freundschaft $\left(, \varphi \imath \lambda i \alpha^{\prime)}\right)$ angesiedelt, welche zwischen Mann und Frau von Natur aus bestehe; im Gegensatz zu den übrigen Lebewesen aber vereinigten sich die Menschen nicht nur um der Nachkommenschaft willen, sondern auch, um die unvermeidlichen Schwierigkeiten des Alltags zu bewältigen. Denn von Anfang an seien die Aufgaben aufgeteilt und für den Mann und die Frau jeweils verschieden; andererseits aber unterstütze man sich gegenseitig, indem man das Eigene jedes einzelnen zum Gemeinsamen beider mache. Deshalb scheine in einer solchen Freundschaft sowohl das Nützliche als auch das Angenehme einen Platz zu haben; die Freundschaft sei darüber hinaus auch tugendgemäß, wenn die Partner entsprechend tugendhaft seien ${ }^{14}$.

Aristoteles scheint sich also insofern von Xenophon zu unterscheiden, als er - in Úbereinstimmung mit seiner insgesamt umfassenderen Sicht, die nicht wie die Xenophons rein utilitaristisch ist - die Gemeinschaft der Ehegatten nicht streng auf die materiellen Güter beschränkt, sondern ihr die Funktion gegenseitiger Hilfeleistung im allgemeinen (unter Umständen auch im Sinne ethischer Vervollkommnung) zuweist. Die eheliche Gemeinschaft ist also nicht mehr nur ein Mittel zur möglichst nutzbringenden Anwendung des Prinzips der Arbeitsteilung auf die Verwaltung und Vermehrung der Familiengüter in ihrer Gesamtheit, sondern auch eine Form der Verwirklichung jener vielschichtigen Beziehung , $\varphi \imath \lambda i \alpha^{\prime \prime}$; insofern aber entzieht sie sich - noch mehr als bei Xenophon - jedweder juristischen Qualifikation, ist doch die Art und Weise ihrer Verwirklichung ganz von den konkreten Lebensumständen und eventuellen Erfordernissen der Hilfeleistung abhängig ${ }^{15}$.

An anderer Stelle bemerkt Aristoteles, daß die Ehefrau bisweilen, besonders wenn sie eine $\varepsilon \pi i k \lambda \eta \rho \circ \zeta$ ist, zum Befehlen neige; er brandmarkt dieses Verhalten und vergleicht es mit dem in Oligarchien zu beobachtenden Phänomen, daß Ämter nicht nach Qualifikation, sondern aufgrund von Reichtum und Macht vergeben werden ${ }^{16}$. Noch deutlicher als bei Xenophon ergibt sich daraus, daß ein etwaiger größerer Reichtum der Frau dem Ehemann keinesfalls seine Vorrangstellung „bei dem, was ihn an-

\footnotetext{
14 Arist. EN VIII,1162a, 16-29. Eine deutsche Úbersetzung bei Gaiser, Ehe 29-30. Zur Aufgabenteilung vgl. auch Arist. EN VIII,1160b, 34-35.

${ }_{15}$ An anderer Stelle (Pol. II, 5,1262b,37-1263a,40) ist sich Aristoteles (im Rahmen einer Diskussion der verschiedenen Eigentumsformen innerhalb der städtischen Verfassung) der Unterschiede zwischen juristischem Miteigentum, einfacher Gebrauchsgemeinschaft und komplexeren Konstruktionen völlig bewußt; ebenda 1263a, 29-30 interpretiert er das Sprichwort „Kotvò tò

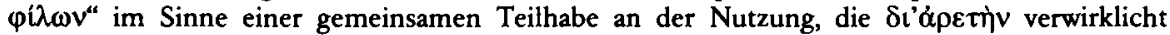
werde. Dies bedeutet jedoch nicht, daß sich die eheliche Gemeinschaft allein auf diesen Aspekt beschränkt. Eine Zusammenstellung der überaus zahlreichen Stellen, welche die Verbreitung des zitierten Sprichwortes (laut D.L. VIII,10 pytagoreischer Herkunft) bezeugen, in: Aristote. L'éthique à Nicomaque, introduction, traduction et commentaire par René Antoine Gauthier et Jean Yves Jolif (Louvain-Paris ${ }^{2}$ 1970) II,2,697, ad Arist. EN VIII,1159b,31.

16 Arist. EN VIII,1161a, 1-3.
} 
geht", streitig machen darf, und ebendies impliziert offensichtlich, daß er über das Vermögen seiner Frau verfügen kann. Im übrigen wird die Vorrangstellung des Mannes in der Ehe, welche in Xenophons Oikonomikos zwar auch faktisch bestand (war es doch Ischomachos, der seine Frau belehrte und ihr Richtlinien für die Haushaltsführung gab), nicht aber offen in Form einer Vorschrift zum Ausdruck gebracht wurde ${ }^{17}$, von Aristoteles mehrfach ausdrücklich ausgesprochen: Die Freundschaft unter den Eheleuten beruhe nicht auf der Gleichberechtigung der Partner, sondern auf der Úberlegenheit des Mannes. Die Gemeinschaft unter Ehegatten sei ihrer Natur nach aristokratisch, selbst wenn (um der Gefahr eines Abgleitens in die Oligarchie vorzubeugen) der Ehefrau in den sie betreffenden Bereichen ein eigenständiger Wirkungskreis zuzuerkennen sei ${ }^{18}$; der Ehemann regiere seine Ehefrau wie der Magistrat eine Stadt $^{19}$. Daraus folge, daß die Tugenden des Mannes und der Frau nicht dieselben seien $^{20}$ (im Gegensatz zu der diesbezüglichen Ansicht des Sokrates ${ }^{21}$ ) und daß folglich die jeweiligen Tätigkeiten nicht nur in verschiedenen Bereichen entfaltet würden, sondern auch von unterschiedlicher Bedeutung seien: Der Mann sei seiner Natur nach eher zum Führen befähigt als die Frau ${ }^{22}$, weil in dieser die rationale Fähigkeit schwach sei, wenn sie ihr auch nicht abgehe wie dem Sklaven oder wenn sie auch nicht so unvollständig entwickelt sei wie beim $\mathrm{Kinde}^{23}$.

Daraus kann leicht ersehen werden, daß es grundsätzlich im eigenen Interesse der Frau liegt, sich dem Manne überall dort, wo ihm die Führung zukommt, unterzuordnen $^{24}$, und dies kann natürlich nicht ohne Auswirkungen auch auf die Verwaltung des Vermögens bleiben. Xenophons Bemühungen um eine stärkere Gleichberechtigung gehen hier ins Leere.

4. Im ersten Buch des dem aristotelischen Corpus angehörenden, allgemein aber einem Schüler zugeschriebenen Oikonomikos wird der Gedanke der Nikomachischen Ethik und der Politik wiederaufgenommen, daß die Ehegemeinschaft nicht nur die Erhaltung des Menschengeschlechts bezwecke, sondern auch das Wohlergehen der Ehepartner, welche von Natur aus für die Vereinigung miteinander bestimmt seien. Der Autor bezieht sich hier auf die Auffassung Xenophons, der dem Mann im wesentlichen eher aktive Erwerbstätigkeiten zugewiesen hatte, der Frau dagegen eher passive Obhutsaufgaben ${ }^{25}$.

17 Vgl. allerdings X. Oec. III, 10-15; Smp. II,9.

18 Vgl. jeweils Arist. EN VIII,1158b,11-14; 1160b,32-36; 1161a,22-25.

19 Arist. Pol. I,12,1259b,1, vgl. 1254b,14-15.

20 Arist. EN VIII,1158b,18; Pol. I,13,1259b-1260a.

${ }^{21}$ Zum sokratischen Denken vgl. Arist. Pol. I,13,1260a, 22; X. Smp. II,9; Eva Cantarella, L'ambiguo malanno (Rom ${ }^{2} 1985$ ) 81-84. Úber die Stellung Platos vgl. beispielsweise Eva Cantarella, a.O. 87-89; Silvia Campese, La donna e i filosofi, in: Associazione italiana di cultura classica. Atti del convegno nazionale di studi su La donna nel mondo antico, Torino 21-22-23 Aprile 1986, a cura di Renato Uglione (Torino 1987) 106-112.

22 Arist. Pol. I,5,1254b,13-14; I,12,1259b,2-3; VII,3,1325b,3-4.

23 Ibid., I,13,1260a,12-13.

${ }^{24}$ Ibid. III,6,1278b,37-39.

25 Ps.-Arist. Oec. I,3,1343b,18-1344a,7. 
Der selbstāndige Bereich der Frau wird genauer im 3. Buch umschrieben (welches wahrscheinlich erst erheblich später entstand als das 1. und lediglich in mittelalterlichen lateinischen Übersetzungen überliefert ist): Ihr soll die Leitung der hausinternen Angelegenheiten obliegen (um die sich zu kümmern für den Mann nicht schicklich ist), und im besonderen soll sie die Kontrolle über die Ausgaben für Feste ausüben, wobei die durch die Gesetze der Stadt bestimmten Beschränkungen zu beachten sind. In allem anderen aber soll sie ihrem Gatten gehorsam sein und sich seinen Gewohnheiten anpassen ${ }^{26}$. Gedanken des 1 . Buches aufgreifend und weiterentwickelnd, wendet sich der Autor mit zahlreichen Ermahnungen an den Ehemann, wobei im vorliegenden Zusammenhang von besonderem Interesse ist, daß ihm empfohlen wird, durch Treue, Umsicht und Unterweisung ein so harmonisches Verhältnis zu seiner Frau zu entwickeln, daß diese im Falle seiner Abwesenheit die gemeinsamen Belange so wahrnehmen könne, als ob er anwesend sei $^{27}$. Kurz darauf zitiert und erläutert der Autor die Verse Homers, daß es nichts Besseres und Erhabeneres gäbe als einen Ehemann und eine Ehefrau, welche in voller Willensübereinstimmung den Haushalt führ$\operatorname{ten}^{28}$. Schließlich werde sich jeder um die Eltern des anderen kümmern müssen sowie um die Kinder, die Freunde, die Güter und das ganze Haus als gemeinsames Vermögen ${ }^{29}$.

In dieser ganzen Abhandlung bleiben allerdings die Einzelheiten der Gütergemeinschaft im Hintergrund - genau wie in den authentischen Werken des Aristoteles. Während aber das erste Buch des Oikonomikos noch eine inhaltliche Verbindung zwischen den Vorstellungen des Aristoteles und dem Modell Xenophons herzustellen suchte, scheint sich im dritten Buch die Perspektive allmählich zu verändern, indem nunmehr der moralische und geistige Aspekt der Ehegemeinschaft in den Vordergrund tritt und als notwendige Voraussetzung auch der wirtschaftlichen Komponente erscheint; dabei wird die vergleichsweise klare Scheidung von Wirkungsbereichen der Ehegatten zugunsten der Darstellung eines Idealzustandes aufgegeben, der die Frau dazu veranlaßt, sich die Wünsche und Bedürfnisse des Mannes in einem solchen Ausmaß zu eigen zu machen, daß sie notfalls imstande wäre, an seiner Stelle und in seinem Sinne das Familienvermögen zu verwalten (übrigens ein Thema des Stoikers Antipatros, an dessen Einfluß man hier deshalb denken könnte). Daß der Autor so nachdrücklich die Bedeutung des Verhaltens des Ehegatten für eine solche Úbereinstimmung in Absichten und Zielen hervorhob, legt die Annahme nahe, daß es seiner Ansicht nach in erster Linie dem Manne als dem Hauptverantwortlichen für das Familienvermögen zukam, dafür zu sorgen, daß die Frau es übernahm, sich darum wie

\footnotetext{
${ }^{26}$ Ps.-Arist. Oec. III,1,140,6-141,11 Rose.

${ }^{27}$ Ibid. III,3,145,2-5 Rose: „Ex hiis quoque omnibus eligens meliora, uxorem sibi concordem et fidelem et propriam facere decet, ut praesente viro et non utatur semper non minus ac si presens adesset, ut tamquam rerum communium curatores...". Möglicherweise liegt hier ein Anklang an die aristotelische Lehre vor, die den Freund als alter ego bezeichnet: Arist. EE VII,1245a,30-31; EN IX,1166a,31-32.

${ }^{28}$ Ps.-Arist. Oec. III,4,146,17-19 Rose: „Nihil enim maius bonum ipsa in hominibus ait esse quam cum concordes vir et uxor in voluntatibus domum regunt"; vgl. Hom. Od. VI,181-185. 29 Ibid. III,4,147,5-11.
} 
um ihr eigenes Vermögen zu kümmern ${ }^{30}$. Es bleibt die Tatsache, daß sich die Argumentation auch hier auf der Ebene der Verwaltungstätigkeit bewegt, wir uns also nicht auf dem Niveau einer einfachen Gebrauchsgemeinschaft befinden. Andererseits gelangt der Autor, wenn er auch von der minuziösen Aufgabenteilung im Sinne Xenophons ausgeht, schließlich zu dem - wenn auch noch ziemlich verworrenen - Denkmodell einer Art gegenseitiger Austauschbarkeit der Gatten bei der Verwaltung des Vermögens.

5. Dieses letzte Thema wurde ziemlich breit von Antipatros aus Tarsos abgehandelt, welcher um die Mitte des 2.Jahrhunderts v. Chr. Nachfolger des Diogenes von Seleukeia als Haupt der stoischen Schule in Athen wurde. Indem er jungen Leuten eine Eheschließung empfahl, verglich er die Ehe mit den anderen Arten der Freundschaft $\left(, \varphi \imath \lambda i \alpha^{\prime \prime}\right)^{31}$ und unterschied sie dabei - anders als Aristoteles - sehr deutlich von den letzteren: Während nämlich jene der Vermengung von Gegenständen ähnelten, die man nebeneinander stellen könne (wie Linsen oder andere Hülsenfrüchte), stelle die Verbindung der Gatten eine vollständige Vermischung dar, vergleichbar derjenigen von Wein und Wasser. Denn sie allein haben nicht nur das Vermögen, die Kinder und die Seele, sondern auch die Körper gemeinsam. Auch unter einem anderen Ge-

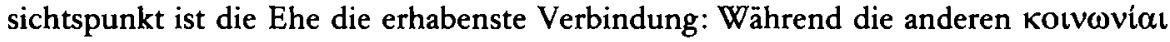
auch andere Zwecke verfolgen, hat sie das Ziel, aus beiden Teilen eine einzige Seele zu formen (es wird allerdings anscheinend erwartet, daß sich vor allem die Frau der Seele des Mannes anpasse) ${ }^{32}$. Nach einer Erwiderung auf mögliche Einwände - hier erfährt man, daß der Mann die Frau die oikovouí $\alpha$ zu lehren habe, die Art und Weise, das Haus gedeihen zu lassen, sowie den Sinn und den Zweck der ehelichen Gemeinschaft und auch noch die religiösen Pflichten und die sittlichen Verhaltensregeln - zählt Antipatros die Vorteile einer harmonischen Vereinigung auf: Es werde dadurch einfacher, die Beschwernisse des Lebens zu meistern - ganz ähnlich wie für jemanden, der nur eine Hand oder nur einen Fuß habe und dann den fehlenden Körperteil bekomme. Da die Ehegatten nun nicht mehr allein, sondern zu zweit seien, verdoppelten sich ihre Fähigkeiten; jeder von ihnen habe ein alter ego, das ihn unterstützen könne. Dies sei besonders wichtig, wenn sich ein Mann der Philosophie oder der Politik widmen wolle; in diesem Falle bedürfe er nämlich erst recht einer Ehefrau, die für ihn die Verwaltung übernehme.

In unserem Zusammenhang sind es im wesentlichen drei Aspekte, die Antipatros besonders hervorhebt: die das gesamte Leben umfassende Natur der Ehegemeinschaft

30 In ganz anderem Sinne (nämlich eines Anspruches der Frau, im Hause des Mannes zu herrschen) hātte sich Theophrastos geãußert, wenn das - wohl über Seneca, De matrimonio - von Hieron. Adv. Iovin. I,47(314B-C) (ed. E. Bickel, Diatribe in Senecae philosophi fragmenta. I. Fragmenta de matrimonio, Lipsiae 1915,389) gelieferte Bild stimmt. Jedenfalls erwähnt er die Gütergemeinschaft nicht.

${ }^{31}$ Stob. IV,22,25 (= Flor. 67,25), ed. Hense IV,507-512 = Ioannes ab Arnim, Stoicorum veterum fragmenta (Lipsiae 1903) III,254-257, Fr. 63. Eine deutsche Übersetzung bei Gaiser, Ehe, 36-39.

${ }^{32}$ Der Text ist hier leider verdorben, und der Sinn hängt weitgehend von der gewählten Korrektur ab: vgl. Stob. ed. O. Hense, IV,508,20, App. 
(von der die Gütergemeinschaft nur ein Bestandteil sei), ihr Ziel der Formung einer einzigen Seele und die Ausbildung der Fähigkeit der Frau, notfalls auch selbständig das ganze Familienvermögen zu verwalten, wenn nämlich der Mann anderen Beschäftigungen nachgehen müsse. (Hier wird die Vorstellung vom Ehegatten als alter ego, die von Aristoteles übernommen wurde, der sie allerdings in bezug auf den Freund äuBerte $^{33}$, gerade durch die Differenzierung zwischen der Ehe und den anderen Arten der Freundschaft besonders eindringlich.) Diese Gedanken des Antipatros legen es nahe, daß durch sie das dritte Buch des pseudo-aristotelischen Oikonomikos beeinflußt wurde (und nicht etwa umgekehrt).

6. Mit diesen Ansichten fand Antipatros Nachfolger in der stoischen Schule: im 1. Jahrhundert n. Chr. den Römer Musonius Rufus und im 2. Jahrhundert n. Chr. Hierokles von Alexandreia.

Für Musonius ist es der Zweck der Ehe, zusammenzuleben, Kinder zu zeugen und alles als gemeinsames Gut anzusehen und nichts - nicht einmal den Körper - als eigenes Gut jedes einzelnen; dabei seien nicht die Paarung und die Zeugung von Kindern die eigentlichen Merkmale der Ehegemeinschaft, sondern die vollständige Teilhabe am Leben des Partners (was auch eine Gemeinschaft in bezug auf Absichten und Wünsche miteinschließe) und die gegenseitige Fürsorge; wo es daran fehle und sich die eheliche Verbindung auf das Zusammenleben beschränke, werde die Kotvovía notwendigerweise zerstört und verschlechtere sich die Lage der Gatten ${ }^{34}$. Daher sei

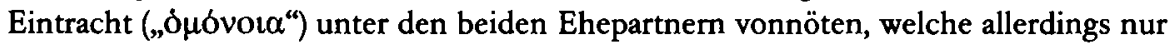
dann bestehen könne, wenn die Gatten tugendhaft seien ${ }^{35}$. Was die Tugendhaftigkeit angehe, seien an den Mann und die Frau gleich hohe Anforderungen zu stellen. Auch hinsichtlich der Arbeitsteilung sei es - selbst wenn einige Aufgaben eher dem Manne, andere eher der Frau zukämen - durchaus zweckmäßig, wenn jeder mit den Tätigkeiten des anderen vertraut $\operatorname{sei}^{36}$. An anderer Stelle bekräftigt Musonius, daß es keine notwendigere und innigere kotv $\omega v i \alpha$ gebe als die eheliche, weil in ihr als einziger unter allen sozialen und familiären Beziehungen alles (Körper, Seelen, Güter) als gemeinsam angesehen werde und weil in ihr die $\varphi \imath \lambda i \alpha$ am stärksten ausgeprägt sei. ${ }^{37}$.

Steht Musonius zwar eindeutig in der Nachfolge des Antipatros, so scheint er in bezug auf die Vermögensgemeinschaft doch besonderen Nachdruck darauf zu legen, daß sie gewissermaßen eine innere Einstellung ist, daß sie eher ein erstrebenswertes Ziel des Ehelebens als dessen Voraussetzung darstellt; auch scheint er größeren Wert auf die Liebe und die gegenseitige Hilfeleistung als auf die Unterscheidung der jeweiligen

${ }^{33}$ Vgl. supra A. 27; Xenophon hielt es für möglich, als alter ego einen entsprechend ausgebilde-

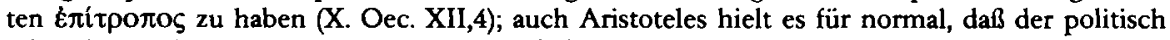

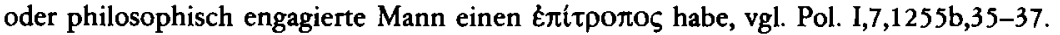

${ }^{34}$ Stob. IV,22,90 (= Flor. 69,23), ed. O. Hense IV,530-531 = C. Musonii Rufi reliquiae, ed. O. Hense (Lipsiae 1905), 67-68, Fr. XIII/A.

35 Stob. IV,22,104 (= Flor. 70,14), ed. Hense IV,540= Muson. Fr. XIII/B ed. Hense 69-70.

${ }^{36}$ Stob. II,31,123 = Muson. Fr. IV, ed. Hense 16,15-17,20; dies schließt die Vorrangstellung des Mannes nicht aus: vgl. Fr. VIII ed. Hense 39,17-18.

37 Stob. IV,22,20 (=Flor. 67,20), ed. Hense IV,497-501 (bes. 500,7-9)=Muson. Fr. XIV ed. Hense 70-76 (bes. 74,7-10). 
Tätigkeiten zu legen. So leuchtet hier, wenn auch noch recht unbestimmt, ein ziemlich neues Ehekonzept auf: Statt in einer präzisen Arbeitsteilung (nach dem Modell Xenophons) oder auch in einer vollständigen Austauschbarkeit des Mannes durch die Frau (nach der Vorstellung des Antipatros) verwirklicht sich die Zusammenarbeit der Gatten nach Musonius eher in einem liebevollen Zusammenwirken bei denselben Tätigkeiten und vielleicht auch Entscheidungen.

Dieser Aspekt wird von Hierokles noch stärker betont - selbst wenn bei ihm auch nicht der Hinweis fehlt, daß der Mann $\not \rho \chi \varepsilon^{38}$ : Nachdem er hervorgehoben hat, daß die Ehe nützlich sei (sowohl wegen der Kinderzeugung als auch um anderer Vorteile willen wie etwa demjenigen, daß die Frau während der Abwesenheit des Mannes als Wächterin des Hauses dienen könne), bezeichnet er die Ehe auch als schön ${ }^{39}$; wahre

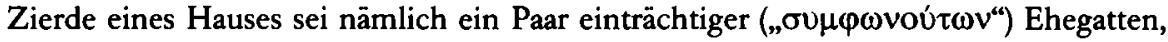
die alles gemeinsam besäßen - einschließlich der Körper, aber vor allem der Seelen und die sich gemeinschaftlich um die Führung des Haushaltes kümmerten, um die Überwachung der Sklaven, die Aufzucht und Erziehung der Kinder sowie um den gesamten Lebensunterhalt. Die Gütergemeinschaft sei folglich ein Mittel zur Herstellung vollstāndiger Eintracht. Es verwundert dabei nicht, daß Hierokles bei der Behandlung der oikovo $\mu i \alpha$ die traditionelle Aufgabenteilung zwischen Mann und Frau sehr wohl kennt, zugleich aber einerseits Wert auf die Feststellung legt, daß es auch Hausarbeiten gäbe, die dem Manne zukämen (wie Holzhacken, Wasserschöpfen, Mahlen, Kneten des Brotteiges), andererseits auf die Betonung der (auch schon von Musonius hervorgehobenen) Notwendigkeit, daß jeder mit den Tätigkeiten des anderen vertraut sein müsse, weil dies die ehelichen Bande festige ${ }^{40}$.

7. Ungefähr zur gleichen Zeit wie diese stoischen Schriften (die, wie gezeigt, durch das Ideal einer - in erster Linie als geistige Einstellung aufgefaßten - „vollständigen Gemeinschaft" charakterisiert sind) entstand ein blühendes neupythagoreisches Schrifttum. Unter diesen Werken, die häufig fiktiven Autoren zugeschrieben sind und deren Lokalisierung (nach Alexandreia, Rom oder Süditalien) sowie Datierung (zwischen dem 3. Jahrhundert v. Chr. und dem 2. Jahrhundert n. Chr.) ganz unsicher sind, sind in diesem Zusammenhang vor allem zwei Schriften ziemlich unterschiedlicher Ausrichtung von Interesse.

Da sind zunächst die unter dem Namen eines Kallikratidas überlieferten Stellen ${ }^{\mathbf{4}}$, welche aristotelische Gedanken wiederaufzunehmen scheinen, wird hier doch hervorgehoben, daß in der Ehe (deren Zweck die Lebensgemeinschaft sei) dem Mann die

${ }^{38}$ Stob. IV,22,23 (=Flor. 67,23) ed. Hense IV,503 = Hans von Arnim, Hierokles' ethische Elementarlehre (Berliner Klassikertexte IV, Berlin 1906), 53,15-16 (im folgenden Arnim, Hierokles). 39 Stob. IV,22,24 (Flor. 67,24) ed. Hense IV,503-507, bes. 505,5-20=Arnim, Hierokles, 53-55, bes. 54,14-25. Deutsche Übersetzung bei Gaiser, Ehe 47-50.

${ }^{40}$ Stob. IV,28,21 (= Flor. 75,21) ed. Hense V,696-699=Amim, Hierokles, 62-63.

${ }^{41}$ Für unsere Thematik sind besonders relevant die von Stobaios IV,28,17-18 zusammengetragenen Stellen (=Flor. 85,17-18) ed. Hense V,684-688= Holger Thesleff, The Pythagorean Texts of the Hellenistic Period (Acta Academiae Aboensis, ser. A, Humaniora, Bd.30,1, Abo 1965), 105,10-107,11 (im folgenden Thesleff, Texts). Ausführlicher Kommentar bei Friedrich Wilhelm, Die oeconomica der Neupythagoreer Bryson, Kallikratidas, Periktione, Phintys. Rheinisches Museum für Philologie, N.F. 70 (1915), 177-181. 
Führung zustehe, welche allerdings „politischer" Natur sei, weil ja auch der Mann an der Kotvwvía teilhabe und die Führung daher im gemeinsamen Interesse auszuüben habe. Auch der Hinweis darauf, daß eine Ehefrau, die reicher sei als ihr Mann, dazu neige, eine Vorrangstellung einzunehmen, und daß dadurch Streitigkeiten hervorgerufen würden, erinnert an einen aristotelischen Gedanken. Nicht besonders originell sind auch die Folgerungen: Einerseits sei es sinnvoll, sich eine Frau zu nehmen, die aus ähnlichen wirtschaftlichen Verhältnissen stamme; andererseits müsse er ihr gegen-

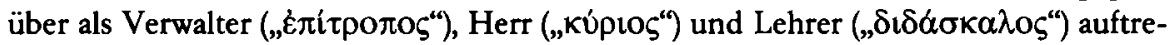
ten, indem er sich um ihr Vermögen kümmere, sie selbst leite und unterweise.

Kallikratidas betont also offensichtlich noch stärker als Aristoteles den Leitgedanken, daß in der Ehe dem Manne die Vorrangstellung zustehe - und sei es auch nur zum gemeinsamen Nutzen; diese äußere sich in verschiedenen Befugnissen, zu denen auch die Verwaltung der Güter der Gattin gehöre (wobei die Mitgift nicht von etwaigen anderen Vermögensgegenständen der Frau unterschieden wird). Die Aufgaben der Frau werden nicht näher umschrieben, verbleiben gewissermaßen im Dunkel blinden Gehorsams dem Manne gegenüber. Allerdings könnte dies auch auf den fragmentarischen Zustand der Überlieferung zurückgehen; denn in anderen neupythagoreischen Schriften wird die Arbeitsteilung zwischen Mann und Frau in Worten beschrieben, die dem Sinne nach den Vorstellungen Xenophons recht nahe kommen ${ }^{42}$.

8. Unter diesen Werken ist am vollstāndigsten überliefert (und insofern einem Gesamturteil zugänglich) ein Oikovoutкós, der dem Philosophen Bryson (einem Schüler des Pythagoras) zugeschrieben ist und der - abgesehen von einigen Fragmenten bei Stobaios - in einer vielleicht aus dem 10.Jahrhundert stammenden arabischen Übersetzung erhalten ist, welche dann ihrerseits einer lateinischen Epitome und einer hebräischen Fassung als Vorlage diente ${ }^{43}$. Der Verlust des Originaltextes gebietet Vorsicht bezüglich der Beurteilung von Einzelheiten; dennoch kann man hier einen interessanten Versuch erkennen, die Menge der in früheren Schriften herausgearbeiteten Elemente in einem einzigen Gesamtbild zusammenzuführen und in eine strenge Ordnung zu bringen.

Dabei geht der Autor vom Zweck der Ehe aus, der einerseits durch die Natur bestimmt sei (Zusammenwirken von Mann und Frau bei der Hervorbringung von Nachkommenschaft), andererseits aber auch durch die Vernunft: Da der Mann ja um des Lebensunterhaltes willen sein Haus verlassen müsse, brauche er eine Person, die es für

42 Vgl. hauptsächlich Phintys, in Stob. IV,23,61 (=Flor. 74,61) ed. Hense IV,588-591 (bes. 589,3-17) = Thesleff, Texts, 151-153, wo die Diskussion der Aufgabenteilung mit derjenigen der weiblichen Tugenden verbunden ist; im Vergleich zu Xenophon fehlt allerdings eine konkrete Erwähnung der Gütergemeinschaft. Vgl. auch Wilhelm, a.O. 208-212. - Andere neupythagoreische Schriftsteller betonen - wie Kallikratidas (und das 3. Buch des pseudoaristotelischen Oikonomikos) - die Notwendigkeit, daß sich die Frau nach dem Willen und den Wünschen des Mannes richte: vgl. Melissa in Thesleff, Texts 116,11-15; Periktione in Stob. IV,28,19 (= Flor. $85,19)$ ed. Hense V,691,14-16 und $692,8-10=$ Thesleff, Texts, 144,8-10;16-18; vgl. Wilhelm, a.O. 199.

${ }^{43}$ Martin Plessner, Der olkovoutkós des Neupythagoreers „Bryson“ und sein Einfluß auf die islamische Wissenschaft (Orient und Antike, 5, Heidelberg 1928) 205-214: Ausgabe der lateinischen Epitome; 214-259: deutsche Ubersetzung des arabischen Textes (im folgenden Plessner). 
ihn hüte und besorge. Da sich jedoch niemand um fremde Angelegenheiten mit der gleichen Sorgfalt wie um eigene kümmere, sei es am besten, einen Partner zu haben, der das Haus in derselben Weise wie der Hausherr als eigenes ansehen könne und aus diesem Grunde den Haushalt mit demselben Eifer wie dieser selber führen könne ${ }^{4 \underline{4}}$. Wie nun die Frau von der Natur zum Zusammenwirken mit dem Manne bei der Kinderzeugung geschaffen sei, so besitze sie die natürliche Befähigung, während der Abwesenheit des Mannes dessen Stelle im Hause einzunehmen, wohingegen umgekehrt der Mann sie in dem ergänzen könne, was ihr abgehe, nämlich durch die Begabung, außer Haus den Lebensunterhalt zu beschaffen. Die Person, deren ein Mann bedürfe, sei also die Ehefrau, zumal die Gottheit zwischen beiden Gefühle der Liebe und der Zusammengehörigkeit entstehen lasse, welche Gefühle des Neides, der Rivalität und der Habsucht in bezug auf die Verwaltung des Vermögens bei ihnen ausschlössen ${ }^{45}$. Kurzum, die beiden seien so beschaffen, als bildeten sie eine einzige Person.

Da der Mann seiner Frau sein eigenes Haus überlasse und sie zur Hausherrin einsetze $^{46}$, müsse sie ihm ergeben sein und sich ihm unterordnen, indem sie seinen Anordnungen Folge leiste; selbstverständlich sei sie von ihm über ihre Aufgaben in bezug auf Mithilfe, Aufsicht und Bewahrung des gesamten Vermögens zu belehren. Kurzum, Familienoberhaupt sei der Mann ${ }^{47}$. Für das Wohlergehen der Familie müßten die Charaktere der beiden Gatten miteinander harmonieren, was voraussetze, daß die Partner tugendhaft seien ${ }^{\mathbf{4 8}}$; die Frau solle die gleichen moralischen Qualitäten wie der Mann besitzen, um ihn bei seiner Vervollkommnung unterstützen und um die Führung des Haushaltes übernehmen zu können ${ }^{49}$; mit einer lebhaften und teilnahmsvollen Beschreibung solcher Tugenden endet das der Frau gewidmete Kapitel in der Schrift Brysons ${ }^{50}$.

Die Ehe wird also wiederum - wie schon bei Xenophon - unter einem rein ökonomischen, mithin recht beschränkten Blickwinkel gesehen, was möglicherweise auch durch das literarische Genus bedingt sein mag; an die Stelle einer minuziösen Aufzäh-

44 Bryson $§ \S 74-76$ bei Plessner, 233-234; lateinische Epitome, ibid. 207-208. - Die Bemerkung, daß man sich um fremde Angelegenheiten nicht mit der gleichen Sorgfalt kümmere wie um die eigenen, findet sich bei Ps.-Arist. Oec. I,6,1344b,35-36. Bereits Aristoteles, Pol. II,3,1261b,33-35, hatte konstatiert, daß man sich intensiv um die eigenen Angelegenheiten, aber wenig um die gemeinsamen kümmere. Die Stellung der Frau als Alter ego des Mannes hob hauptsāchlich Antipatros hervor.

45 Bryson $\$ \S 80-81$ bei Plessner $235 \mathrm{f}$. Der letzte Satz der arabischen Fassung ist zusammenhanglos; verbessert man den Text nicht, so dürfte sich aber ungeführ dieser Sinn ergeben (für eine eingehende Diskussion des schwierigen Passus bin ich Frau Dr. Roberta Aluffi zu herzlichem Dank verpflichtet). Die hebräische Fassung übersetzt hingegen anders: „sondern einer gibt dem anderen freiwillig sein Geld" (Plessner 235, $\mathrm{x}-\mathrm{x}$ ).

${ }^{46}$ Frau Dr. Aluffi wies mich darauf hin, daß der arabische Text den Terminus mallaka verwendet, der „zum Eigentümer machen“ (eventuell auch in juristisch-technischem Sinn) bedeutet.

47 Bryson $\S \S 82-84 ; 87$ bei Plessner 236-237; 238.

48 Bryson $\$ \S 94-95$ bei Plessner 240; es liegt hier eine enge Verwandtschaft mit Muson., Fr. XIII B, ed. Hense $70,1-4=$ Stob. IV,22,104 (=Flor. 70,14) ed. Hense IV 540,20-26 vor; viel vager ist hingegen die Ähnlichkeit mit Arist., De an. 411a,5, auf die Plessner 283 hingewiesen hat.

49 Bryson $\$ \$ 91-93$ bei Plessner 239-240; vgl. $\$ \S 96-97$, ibid. 240-241.

so Bryson $\$ \S 96-103$ bei Plessner 240-242. 
lung der Tätigkeiten einer Hausfrau tritt hier aber eine Darstellung der besonderen Eigenschaften, die zu ihrer Vervollkommnung beitragen und ihr einen der Würde des Mannes entsprechenden Rang verleihen (eine Ansicht, die auch andere - sowohl neupythagoreische wie stoische - Autoren teilten ${ }^{51}$ ). Die Funktion der Frau als alter ego des Ehemannes war, wie wir sahen, im 3. Buch des pseudo-aristotelischen Oikonomikos andeutungsweise und dann wesentlich deutlicher bei dem Stoiker Antipatros von Tarsos beschrieben worden; Bryson ist aber offenbar der erste, der sie in einen strengen Argumentationszusammenhang stellt, in welchem auch die Frage der Gütergemeinschaft zur Sprache kommt: Die Ehefrau könne das für den Mann erforderliche andere Ich sein, weil ihr Mutter Natur die entsprechenden Eigenschaften verliehen habe; aber um dieser Rolle gerecht werden zu können, müsse sie, was den Haushalt und die Güter des Mannes angehe, in dieselbe Stellung versetzt werden, die er einnehme, das heißt - so scheint es - Miteigentümerin werden. Auch hier ist es - worauf schon bei anderen Werken hingewiesen wurde - nicht möglich, den Beweis dafür zu erbringen, daß auf ein Miteigentum im juristisch-technischen Sinne angespielt wird, doch legt die Stringenz der Argumentation ebendiesen Schluß nahe, weil nur so sichergestellt wird, daß die Frau die Vermögensgegenstände ihres Mannes auch wirklich als ihre eigenen ansieht. Aber selbst wenn man (wie es vielleicht richtiger ist) davon ausgeht, daß es nicht die Absicht des Verfassers war, seine Ausführungen in juristischer Hinsicht zu präzisieren, so bleibt es dennoch dabei, daß er der Ehefrau die allgemeine Befugnis einräumt, die Güter des Gatten so zu verwalten, wie ihm dies selbst als Eigentümer zusteht. Diese Befugnis erstreckt sich - zumindest im Falle seiner Abwesenheit - nicht nur (wie bei Xenophon) auf die Führung des Haushaltes, sondern auf die Verwaltung des gesamten Vermögens. Andererseits muß man wohl der Frau, um ihr Interesse an einer gedeihlichen Entwicklung der Vermögensverhältnisse zu wecken, wenigstens die Haushaltsführung nicht nur in Abwesenheit, sondern auch in Anwesenheit des Mannes anvertrauen. Der erste Schritt ist also Sache des Mannes und wird als ein „Miteinander-Teilen“ (nämlich des Hauses) begriffen, dem die notwendige Unterweisung vorauszugehen hat (hier denkt man unwillkürlich an Xenophon).

Hieran knüpft Bryson zwei Überlegungen, die ihm gestatten, einerseits den (aristotelischen) Gedanken der männlichen Überlegenheit und andererseits den (eher stoischen) einer vollständigen geistigen Vereinigung der Ehepartner aufzunehmen. Daraus, daß der Mann der Frau sein eigenes Haus und seine eigenen Güter anvertraut, leitet Bryson die Gehorsamspflicht der Frau - schon aus Dankbarkeit - ab, um dann etwas gewunden - zu der Feststellung zurückzukehren, daß der Mann in der Ehe und in der Familie als das höherwertige Element anzusehen sei; ebendiese Feststellung in Verbindung mit dem Umstand, daß es ja immer Aufgabe der Frau ist, den Haushalt zu führen, erlaubt dem Autor die Bemerkung, daß es für ein reibungsloses Zusammenleben des Paares unerläßlich ist, daß die Charaktere der beiden Partner miteinander in Einklang stehen, was nur möglich sei, wenn beide tugendhaft seien. Daraus ergibt

51 Vgl. beispielsweise Phintys (supra, A.42); Muson. Fr. III-IV ed. Hense 8-13 und 14-16 = jeweils Stob. II.31,26 und 123. Ähnlich war aber schon die Haltung Sokrates' und teilweise vielleicht auch Platons, vgl. supra A. 21. 
sich, daß für eine gute Verwaltung des Familienvermögens eine wechselseitige geistige Durchdringung der Gatten erforderlich ist, und zu diesem Zweck muß jeder der beiden einzeln mit Tugenden ausgestattet sein, welche für den Mann und die Frau nicht wesentlich verschieden sind (wodurch eine gewisse Gleichberechtigung hergestellt wird, die an einige - oben behandelte - Aussagen des Musonius erinnert).

Es ist also als Brysons Verdienst anzusehen, daß er versuchte, die Vermögensgemeinschaft in einen logischen Zusammenhang mit der geistigen Gemeinschaft zu bringen und die Notwendigkeit sowohl der einen als auch der anderen nicht ethisch, sondern einfach mit ihrer wirtschaftlichen Zweckmäßigkeit zu begründen.

9. Mögen wir uns auch mit der Erörterung der stoischen und vielleicht auch der neupythagoreischen Auffassung bereits in die Kaiserzeit begeben haben, so dürfen wir doch nicht annehmen, daß diese Ehekonzepte die früheren - und sei es auch nur auf der Ebene der Mentalitäten und der kulturellen Modelle - vollständig verdrängt hätten.

Lassen wir die Auffassung Dionysios' von Halikarnassos außer acht, dessen philosophische Grundannahmen schwer zu bestimmen $\operatorname{sind}^{52}$, so müssen wir darauf hinweisen, daß der Oikonomikos Xenophons im 1.Jahrhundert v. Chr. der römischen Gesellschaft in Ciceros lateinischer Übersetzung nahegebracht wurde; einen Reflex davon darf man vielleicht in der Darstellung der vermögensrechtlichen Beziehungen unter den Ehegatten, die sich in der gemeinhin als laudatio Turiae bekannten Inschrift aus augusteischer Zeit findet, erblicken. Anscheinend hatte die Frau dem Manne ihr eigenes Vermögen anvertraut, damit er sich um die Einkünfte kümmere; andererseits hatte sie die custodia über sein Vermögen übernommen, was wohl - wie bei Xenophon - im Sinne von Haushaltsführung und Ausgabenkontrolle zu verstehen ist ${ }^{53}$; auf

52 Von besonderer Bedeutung ist hier die Stelle Ant. Rom. II,25, wo die (Romulus zugeschriebene) Regelung gelobt wird, nach der die Frau, die mit ihrem Mann die confarreatio vollzogen habe, kotv $\omega v \delta \varsigma$ aller Güter und sacra werde. Es handelt sich dabei weniger um eine Folge der Ehe als um eine solche der conventio in manum. Dionysios verbindet anscheinend eine solche

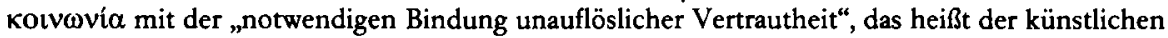
Beziehung agnatischer Verwandtschaft, die sie mit dem Mann und dessen Verwandten einging. Diese Beziehung habe zur Folge, daß sich die Frau gezwungenermaßen nach dem Mann zu richten und ihm in allem zu gehorchen habe. Als Ausgleich dafür werde sie aber kupía des Hauses im gleichen Umfang wie der Mann selbst - und könne zusammen mit den Kindern dessen Erbe antreten. Dionysios hebt - als Ursache dieser Gemeinschaft - die Unauflöslichkeit des ehelichen Bandes hervor (was sicherlich ein neues Element ist), scheint aber zugleich neupythagoreische (auch bei Antipatros begegnende) Themen wiederaufzunehmen, wenn er behauptet, die Frau werde gerade durch den Gehorsam kupi $\alpha$; die kotv $\omega v i \alpha$ wiederum verwirkliche sich auch durch

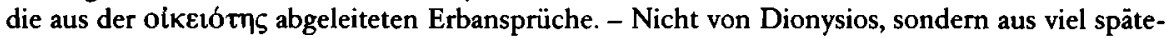
rer Zeit stammt die ihm zugeschriebene Ars rhetorica, wo (II,2-7) Anleitungen für Hochzeitsreden erteilt werden, die für unseren Zweck irrelevant sind.

${ }_{33} \mathrm{Vgl}$. Fontes iuris Romani anteiustiniani. III. Negotia, ed. Vincentius Arangio-Ruiz (Florentiae ${ }^{2} 1969$ ), N.69, S. 209-218 und 625-626; s. auch Erik Wistrand, The so-called Laudatio Turiae (Studia Graeca et Latina Gothoburgensia, 34, Lund 1976). Die fragliche Stelle entspricht I,37-39: „Omne tuom patrimonium acceptum ab parentibus communi diligentia cons[eruauimus]: / neque enim erat adquirendi tibi cura, quod totum mihi tradidisti. Officia [ita par] / titi sumus, ut ego tu[t]elam tuae fortunae gererem, tu meae custodiam sust[ineres...] (Fontes a.O., III,213; ibid. A.1 
diese Weise könnte die gleich darauf folgende Feststellung, daß die Gatten ein gemeinschaftliches Vermögen besäßen ${ }^{54}$, zu verstehen sein.

Ausdrücklich wird dann auf die Ideale Xenophons in einer Schrift des L. Iunius Moderatus Columella Bezug genommen, welcher, wiewohl spanischen Ursprungs, in Rom lebte und dort zur Zeit des Kaisers Claudius bzw. Nero einen Traktat über Landwirtschaft schrieb. Zwar gibt er die traditionelle Ansicht über die Aufgabenteilung der Ehegatten und deren mutmaßlich naturgegebene Ursachen ${ }^{35}$ wieder, doch vermeidet er dabei, die Stellung des Mannes als gewichtiger hinzustellen (so sind die Ausführungen nicht - wie etwa jene des Ischomachos - in Belehrungen, die der Mann seiner Frau erteilt, eingebettet). Erst aus den folgenden Ausführungen, daß nämlich nicht nur in Griechenland, sondern auch in Rom ungefähr bis zur Zeit des Augustus die Hausarbeiten eine fast ausschließliche Aufgabe der matronae gebildet hätten, erfahren wir, daß jene sich mit der ihnen eigenen Emsigkeit bemühten, die „negotia viri“ „maiora atque meliora" zu machen. Dieser Satz ist für die Deutung des folgenden von Bedeutung, wo es heißt, daß im Hause nichts geteilt sei - in dem Sinne, daß der Mann oder die Frau das ausschließliche Eigentum an irgendeinem Gegenstand beanspruchte, daß vielmehr beide in Eintracht "gemeinsam" handelten, wobei die Arbeitsamkeit der Frau den Tãtigkeiten des Mannes außerhalb des Hauses gleichwertig sei ${ }^{56}$. Die (schon xenophontische) Annahme, daß die Hausarbeit der Frau unter wirtschaftlichem Gesichtspunkt der Tätigkeit des Mannes außerhalb des Hauses an Bedeutung durchaus gleichkommen könne, schließt nicht aus, daß es letzten Endes doch das Vermögen des Mannes ist, zu dessen Vermehrung auch die Frau beiträgt. Die "Gütergemeinschaft", die Columella als erstrebenswert ansieht, ist also in dem Sinne zu verstehen, daß der Ehemann der Frau die Führung seines eigenen Haushaltes anvertraut und daß die Ehefrau dieser Aufgabe nachkommt, indem sie sich der von ihrer Familie zur Verfügung gestellten Mittel und Sklaven bedient; natürlich wird der daraus erwachsende Wohlstand - jedenfalls solange die Ehe besteht - auch ihr zugute kommen. Allerdings weist Columella selbst darauf hin, daß dieses Modell schon in seiner Zeit nicht mehr in Geltung war, weil die matronae nunmehr vorzögen, in Luxus zu schwelgen und ein Leben in Muße zu führen, wobei sie es den Sklaven überließen, sich um die häuslichen Angelegenheiten zu kümmern ${ }^{57}$.

Dennoch finden sich zumindest von den Grundlinien der Arbeitsteilung im Sinne Xenophons, wie sie auch von vielen anderen Autoren mehr oder weniger vollständig

finden sich Hinweise auf abweichende Interpretationen; ähnlich deutet den Passus hingegen Wistrand a.O. $39 \mathrm{f}$.); vgl. auch Marcel Durry, Eloge funèbre d'une matrone romaine (Collection des Universités de France, Paris 1950) LXXIVf., wo auf weitere Auslegungsmöglichkeiten hingewiesen wird. S. auch Z.29-33 über die weiblichen Tugenden.

s4 Laud. II,36-37 (Fontes a.O., III,216; Wistrand 26): „... .neque patrimoni no[stri, quod adhuc]/ fuerat commune separa[ti]onem facturam..."; die Stelle könnte allerdings auch auf die von Turias Vater stammende und noch ungeteilte Erbschaft anspielen.

"s Colum. 12 praef., $\S \S 1-6$, wo (in der Übersetzung Ciceros) die Úberlegungen Xenophons (Oec. 7,18-28) dargelegt werden.

s6 Colum., ibid. $\S \S 7-8$.

s7 Colum., ibid. §§ 9-10. 
wiedergegeben werden, noch im 4.Jahrhundert n. Chr. Spuren, z. B. bei Libanios ${ }^{58}$. Letzterer äußert sich jedoch nicht ausdrücklich über den Ausgangspunkt Xenophons, nämlich die Vereinigung der Vermögen in der Absicht, in bezug auf die Gesamtmasse eine Arbeitsteilung vorzunehmen, welche der Natur jedes Partners möglichst entspricht. Aus diesem Grunde können wir hier auf eine vertiefte Behandlung der diesbezüglichen Texte des Libanios verzichten.

10. Ein Autor, der sich (am Ende des 1.Jahrhunderts n.Chr.) zur ehelichen Gemeinschaft äußerte und dabei auch ziemlich ausführlich auf den vermögensrechtlichen Aspekt einging, ist Plutarch. Indem er eine Grundhaltung im Sinne Platons mit typisch stoischen (und teilweise wohl auch neupythagoreischen) Anregungen verknüpfte, kam er zu einer eigenständigen Einstellung, welche durchaus nicht origineller Akzente entbehrt.

Zunächst unterstreicht er die Bedeutung der Liebe unter den Ehegatten, welche er in dem Bemühen um eine Verschmelzung der Seelen verwirklicht sieht, da doch die Ehegatten „weder zwei sein wollen noch glauben, es zu sein"59. Dies äußere sich vor allem in der gegenseitigen Teilhabe nicht nur an den Wechselfällen des Lebens, sondern auch an den Gedanken und Gefühlen - etwa augenblicklicher Freude oder Traurigkeit ${ }^{60}$. Wie ein einheitlicher Gegenstand sowohl einem zusammengesetzten wie auch einem aus verschiedenen Bestandteilen gebildeten entgegengesetzt sei, so bildeten zwei Partner, die sich liebten, ein einziges Wesen, in dem sich die Individuen auflösten wie in einer Mischung von Flüssigkeiten; in diese Verbindung seien sogar die Körper der Gatten miteinbezogen, die Güter, die Freunde und die Angehörigen ${ }^{61}$. Und wie die Natur durch die Vereinigung der Körper ein neues Wesen entstehen lasse, in welchem sich Merkmale beider Elternteile fänden, ohne daß es möglich sei, den Anteil jedes einzelnen wieder zu trennen oder auch nur oberflächlich zu unter-

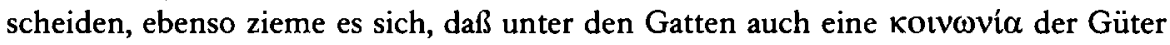
bestehe, daß alles zu einem einzigen Vermögen vermischt und verschmolzen werde, so daß keiner von beiden mehr einen Teil des Vermögens als eigenen und einen anderen Teil als fremden ansehe, sondern daß beide Partner alles als eigen und nichts als fremd betrachteten. Allerdings müßten, wie eine Mischung üblicherweise als "Wein“ bezeichnet werde, selbst wenn sie zum größeren Teil aus Wasser bestehe, auch das

${ }^{58}$ Lib. Thes. XIII,14-15 (ed. Förster VIII,555). Der Mann erwirbt Güter, indem er sich auf dem Forum aufhält; diese Güter übernimmt und bewahrt die Frau zu Hause. Die Sklaven hingegen würden sie vergeuden und somit die Arbeit des Herrn zunichte machen. Dies gilt insbesondere dann, wenn der Mann verreist ist oder einer öffentlichen Tätigkeit nachgeht. - Zuvor ( $(13)$ hatte Libanios bemerkt, daß der Reiche sein Vermögen durch die Ehe verdoppele, der Arme immerhin etwas erwerbe oder jedenfalls aus einer guten, besonnenen und fleißigen Ehefrau großen Nutzen ziehe. Zumindest implizit wird hier also Xenophons Eheauffassung beibehalten.

s9 Plu. Amat. 21,767E; vgl. 770A-B.

${ }^{60}$ Plu. Con. praec. 14,140A; 20,140E; vgl. Brut. 13,7,989f; Dio. 21,7-8, $966 \mathrm{f}$.

${ }^{61}$ Plu. Con. praec. 34,142E-143A. (Plutarchi Moralia Bd. I, recc. et emendd. W. R. Paton et I. Wegehaupt, Leipzig ${ }^{2}$ 1974). Vgl. auch Plu. Amat. 24,769 F. 
Vermögen und der Haushalt als dem Manne gehörend angesehen werden, auch wenn die Frau mehr als der Mann in die Ehe eingebracht habe ${ }^{62}$.

Plutarch nimmt also den stoischen Gedanken einer vollständigen Gemeinschaft unter den Ehegatten wieder auf, geht aber eindringlicher auf ihre vermögensrechtlichen Konsequenzen ein. Auch in dieser Hinsicht müsse gelten, was Platon in bezug auf die $\pi$ ó $\lambda$ ıৎ sage, daß nämlich „das Meinige und das Nicht-Meinige“ nicht auf die einzelnen Individuen, sondern auf die gesamte Gemeinschaft bezogen werden solle ${ }^{63}$. Das Ehepaar werde also ein einziges Vermögen haben, welches jeder der beiden als eigenes empfinden solle, und zwar nicht im Sinne einer Teilhaberschaft nach bestimmten Quoten, sondern in demjenigen einer Berechtigung an dem Ganzen, so als ob das Vermögen zur Gänze einem selbst gehörte (selbst wenn es gleichzeitig vollstāndig auch dem Gatten zustehe) ${ }^{64}$. Plutarch scheint hier auf eine Form des Miteigentums Bezug zu nehmen, die sowohl dem altgriechischen als auch dem römischen Recht bekannt und für Familiengemeinschaften typisch war; vorausgesetzt wird dabei offensichtlich eine größtmögliche Eintracht unter den Gatten, welche so weit geht, daß jeder auch ohne Mitwirkung des anderen den gemeinsamen Willen des Paares zum Ausdruck bringen kann. Es taucht hier wieder ein stoischer Gedanke auf: In der Ehe ist jeder das Alter ego des Gatten und kann ihn daher bei der Verwaltung des gesamten Vermögens vollständig ersetzen.

Plutarch legt jedoch keinen allzu großen Wert auf diesen Gesichtspunkt (den im übrigen schon Antipatros und Bryson eher im Hinblick auf die Frau betont hatten, welche nämlich im Falle der Abwesenheit oder Verhinderung des Mannes als dessen „Ersatz“ fungiere); wichtiger ist ihm vielmehr, daß die Gatten einvernehmlich handeln. Dementsprechend komme ihre Gemeinschaft nicht so sehr in einer Aufgabenteilung zum Ausdruck (über die sich Plutarch - zumindest in seinen Schriften über die Ehe - gar nicht äußert), sondern eher darin, daß sie bei jeder Handlung gleichen Sinnes seien ${ }^{65}$. Es ist indes eine Einschränkung zu beachten: Noch im selben Satz verspürt der Autor das Bedürfnis der Klarstellung, daß das gegenseitige Einvernehmen

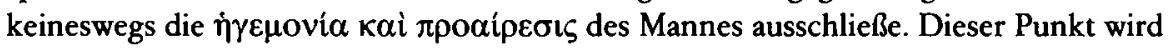
sogar mehrmals hervorgehoben ${ }^{66}$; im übrigen werden, wie wir gesehen haben, auch der Haushalt und das Vermögen dem Manne zugeschrieben, selbst wenn die Frau vielleicht einen größeren wirtschaftlichen Beitrag leistete.

Ein weiterer Hinweis ermöglicht es uns, den Sinn der von Plutarch empfohlenen Gemeinschaft noch genauer zu erfassen. An der Stelle der Coniugalia praecepta, an

62 Plu. Con. praec. 20,140E-F. - Auch andernorts hebt Plutarch eine gewisse Vorrangstellung des Mannes hervor, ohne sie aber zu begründen: vgl. beispielsweise Con. Praec. 8,139B; 11,139D; 19,140D; 33,142E.

${ }^{63}$ Plu. con. praec. 20,140D-E; vgl. auch Amat. 21,767D. Verwiesen wird auf PI. R. V,10,462C.

64 Plu. Con. praec. 20,140F.

65 Vgl. besonders Plu. Con. praec. 11,139C-D. Von Bedeutung auch Qu. Rom. 30,271E, wo der berühmte, von den römischen Gatten ausgesprochene Satz „ubi tu Gaius ego Gaia“ als bezeich-

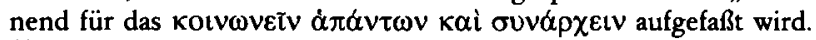

66 Vgl. supra A. 62. Plutarch legt Wert auf die Feststellung, daß der Ehemann sich nicht als Herr,

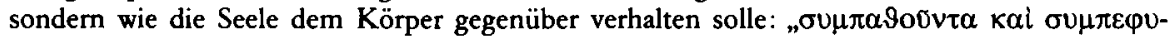

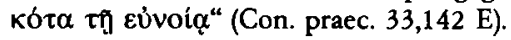


der er die Ehe mit der Vermischung zweier Flüssigkeiten vergleicht, fügt er nämlich hinzu, daß der römische Gesetzgeber verboten habe, daß die Ehegatten einander Geschenke machten oder voneinander erhielten, und zwar nicht, damit sie nichts miteinander teilten, sondern damit sie alles als gemeinsam ansähen ${ }^{67}$. Plutarch will hier offenbar einen möglichen Einwand vorwegnehmen: daß nämlich die von ihm geschilderte eheliche Gütergemeinschaft nicht den römischen Gesetzen entspräche, die ja Schenkungen unter Ehegatten untersagten. Es steht deswegen für ihn außer Frage, daß man die Ehegemeinschaft auch mit rechtlichen Mitteln zu verwirklichen habe, zu welchen etwa die wechselseitige Schenkung des jeweiligen Vermögens gehören könne. Wie aber sei dann das von den römischen Gesetzen aufgestellte Hindernis zu überwinden? Plutarch läßt sich nicht erschüttern und kehrt die Argumentation um: Seiner Meinung nach widerspricht das Verbot gegenseitiger Schenkungen keineswegs der ehelichen Vermögensgemeinschaft, ja es fördere sie sogar. Dieser offensichtlich paradoxen Behauptung liegt etwa folgender Gedankengang zugrunde: Wenn man von "Schenkungen“ spreche, so lege man die Unterscheidung in "mein" und „dein" auch in der Ehe zugrunde und setze für das gemeinschaftliche Eigentum an den Gütern eine Rechtshandlung, aber auch die Möglichkeit einer Beschränkung voraus; da nun aber eine Ehe ohne irgendeine wirtschaftliche Gemeinschaft nicht denkbar sei, müsse das Schenkungsverbot geradezu als ein Ansporn aufgefaßt werden, dafür zu sorgen, daß sich das gemeinschaftliche Eigentum auf alle Vermögensgegenstände der Partner erstrecke, andererseits daß es auf die andauernde gegenseitige Zuneigung gestützt und dementsprechend vom Miteigentum im juristischen Sinne losgelöst werde, weil dieses nämlich zur Geltendmachung egoistischer Forderungen, die sich auf die Unterscheidung zwischen „mein“ und „dein“ gründeten, verleiten könnte.

Notfalls verzichtete Plutarch also anscheinend darauf, sich auf konkrete juristische Bestimmungen zu beziehen, wie dies ja auch bei den anderen behandelten Autoren der Fall war. Es bleibt aber beachtenswert, daß derjenige, der sich an seine Ausführungen hätte halten wollen (welche, wie wir sahen, nicht weit von den wenige Jahre später geäußerten Ansichten des Stoikers Hierokles entfernt sind, die ihrerseits schon von dem Römer Musonius Rufus angedeutete Auffassungen aufgreifen), mit dem Ehepartner wenigstens die wichtigsten die Vermögensverwaltung betreffenden Urkunden hätte aufsetzen müssen, wenn er wollte, daß das Vermögen, obwohl nur ihm juristisch zustehend, fortan als Familiengut angesehen wurde. Dies erinnert an eine einzigartige Beteuerung in der sechsten Satire Juvenals: Der Ehemann, der seine Frau liebt, wird in jedem Fall Qualen erleiden, selbst wenn seine Liebe erwidert wird; denn gegen den Willen seiner Frau wird er kein Geschenk machen können, nichts verkaufen oder kaufen können; in seinen Freundschaften wird er beschränkt sein und sogar bei der Errichtung seines Testamentes ${ }^{68}$.

67 Plu. Con. praec. 34,143A. Im 2. und 3.Jahrhundert n. Chr. debattierten die römischen Juristen über die Gründe, die zu dem Schenkungsverbot unter Eheleuten geführt hatten: vgl. D. 24,1,13 pr.

${ }^{68}$ Iuv. 6,206-218. 
Dies mag indirekt bestätigen, daß das „Modell“ Plutarchs in Rom nicht unbekannt war $^{69}$ und daß man zum Beispiel die Zustimmung beider Gatten zu Veräußerungen von Gegenständen, die rechtlich vielleicht nur einem von beiden gehörten, für erforderlich hielt. Wenn man tatsächlich mit einer solchen Willensübereinstimmung der Partner rechnen muß, so könnte sie sich in Kaufurkunden niedergeschlagen haben, und zwar auch unabhängig von der jeweiligen juristischen Berechtigung. Man wird diesen Gesichtspunkt bei der Interpretation von Dokumenten der Rechtspraxis sowie von Reskripten zu berücksichtigen haben, worauf wir uns hier allerdings nicht weiter einlassen können.

11. Zu Beginn des 5.Jahrhunderts n. Chr. stellte Stobaios einen Großteil der im vorausgehenden erörterten Texte zusammen, aber mit durchaus bedeutungsvollen Ausnahmen: Denn im Abschnitt über die Ehe wird die eheliche Gütergemeinschaft hauptsächlich mit Stellen aus stoischen Schriften (von Antipatros, Musonius und Hierokles) dargelegt ${ }^{70}$; zu diesen Stimmen tritt im nächsten Abschnitt Plutarch hinzu, von dem einige der wichtigsten Stellen wiedergegeben werden - einschließlich des Passus über das Schenkungsverbot unter Ehegatten in den römischen Gesetzen ${ }^{71}$. Dagegen ist Aristoteles nicht berücksichtigt; von Xenophon ist ein Exzerpt über die Begriffsbestimmung der oikovouí (am Ende des diesem Gegenstand gewidmeten Abschnitts $^{72}$ ) aufgenommen, während die Stellen über die Gütergemeinschaft und die Arbeitsteilung der Ehegatten fehlen. An und für sich wird eine solche Aufgabenteilung zwar nicht ignoriert, aber in dem Kapitel, das sich ausführlicher damit beschäftigt

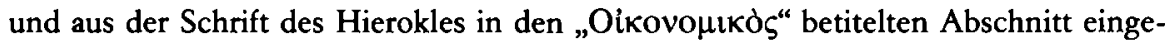
fügt wurde, wird die Arbeitsteilung doch nicht streng entgegengestellt und vor allem dem Erfordernis, die Ehebande zu festigen, untergeordnet, und nicht demjenigen, das Vermögen zu mehren ${ }^{73}$. Die Vorrangstellung des Mannes und die Tugenden der Frau werden hauptsächlich auf der Grundlage neupythagoreischer Texte (von Phintys, Kallikratidas und Periktione) beschrieben ${ }^{74}$; die Vorrangstellung des Mannes aber wird auch in zahlreichen Passagen aus den Schriften anderer Autoren bestätigt ${ }^{75}$.

69 Es ist schwer vorstellbar, daß Juvenal hier auf die Situation anspielt, daß die Frau als Garantie für die Rückgabe ihrer Mitgift eine Hypothek an den Gütern des Mannes vereinbart hatte. Schwer zu bewerten ist auch die ethische und juristische Realitāt bei Martial 4,75. Es könnte sich um eine societas omnium bonorum zwischen Mann und Frau handeln - wie in D. 34,1,16,3 - oder einfach um die Festsetzung einer Mitgift in Höhe des Gesamtvermögens.

${ }^{70}$ Vgl. Stob. IV,22,20 (= Flor. 67,20): Musonius, Fr. XIV Hense; 21-24: Hierokles; 25: Antipatros, Fr. 63 (Arnim, SVF III); alle diese Texte stehen hintereinander im ersten Teil des der Ehe

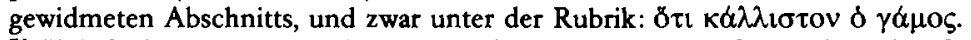

${ }_{71}$ Vgl. Stob. IV,23,43 (= Flor. 74,43), der die ersten zwei Sätze Plutarchs (Con. praec. 20,140 D-E) wiedergibt, während Fr. 44 die beiden letzten Sătze des gleichen Kapitels (140F) reproduziert. Fr. 50 schließlich enthält die Stelle über die Römer (Con. praec. 34,143A). Der ganze Abschnitt trägt die Úberschrift: $\gamma \alpha \mu \iota \kappa \dot{\alpha} \pi \alpha \rho \alpha \gamma \gamma \hat{\varepsilon} \lambda \mu \alpha \tau \alpha$.

${ }^{2}$ Stob. IV 28,23 (=Flor. 85,23) ed. Hense V,701.

${ }^{3}$ Stob. IV,28,21 (=Flor. 85,21) ed. Hense V,696-699; vgl. supra A. 40.

74 Stob. IV,23,61 (=Flor. 74,61): Phintys; IV,28,16-18 (=Flor. 85,16-18): Kallikratidas; 19: Periktione; auch hier lautet die Rubrik: oikovoutkós.

${ }^{5}$ Vgl. beispielsweise Stob. IV,23,5 (=Flor. 74,5): Menander; 20: Philemon; 57 : lamblichos; 58 : Sokrates. 
Will man nun Ioannes Stobaios eine konsequente Einstellung zur Frage der vermögensrechtlichen Beziehungen unter Ehegatten zuschreiben, so müßte man - mit aller Vorsicht, die sowohl durch den anthologischen Charakter seines Werkes als auch durch die Lückenhaftigkeit unserer Untersuchung geboten wird - feststellen, daß er für das Ideal der kotvwví $\alpha$ eintritt, und zwar im Sinne jener (insbesondere von den Stoikern und Plutarch geforderten) vollständigen Gemeinschaft, die sich in erster Linie in einer Geisteshaltung manifestiert. Eine solche Einstellung kann, wenn man sich die damalige Vorrangstellung der neuplatonischen Philosophie vor Augen hält, keineswegs überraschen. Es bleibt aber auf jeden Fall sein Verdienst, das antike Gedankengut den folgenden Jahrhunderten vermittelt $\mathrm{zu}$ haben.

12. Abschließend läßt sich nun festhalten, daß es in der Philosophiegeschichte nicht wenige Autoren gab, die bei der Behandlung der Ehe auch eine vermögensrechtliche Gemeinschaft der Partner für erforderlich hielten. Darunter ist allerdings jeweils Verschiedenes zu verstehen, wobei sich sogar im Lauf der Zeit eine gewisse Entwicklung beobachten läßt, ohne daß aber die jüngeren Wortbedeutungen die älteren vollständig verdrängten.

Wie wir gesehen haben, lassen sich in den behandelten Schriften über die Gütergemeinschaft hauptsāchlich drei Gesichtspunkte auseinanderhalten: a) die Bildung eines einzigen Vermögens, hinsichtlich dessen eine wirtschaftlich sinnvollere, da den jeweiligen Fähigkeiten des Mannes und der Frau eher entsprechende Aufgabenteilung vorgenommen werden soll; b) die Möglichkeit, einen vollwertigen Vertreter zu haben, der die notwendigen Kenntnisse besitzt und die erforderliche Hingabe aufbringt, um das "gemeinsame“ Vermögen als eigenes zu verwalten; c) das ständige Zusammenwirken bei der Verwaltung des ,gemeinsamen“ Vermögens, wobei auch einer allein für beide zusammen handeln kann. Während die beiden letztgenannten Punkte offenkundig eng zusammengehören, stehen sie - zumindest theoretisch - in einem gewissen Gegensatz zum ersten Punkt. Für keinen der drei Aspekte ist indes ein (juristisches) Miteigentum an den Vermögensgegenständen unerläßliche Voraussetzung ${ }^{76}$, selbst wenn dies zur Verwirklichung der ersten beiden Punkte von Nutzen sein könnte, um nämlich ein Interesse an den geforderten Verhaltensweisen zu erwecken. Andererseits beziehen sich alle drei Gesichtspunkte auf die Verwaltung des Vermögens und nicht nur auf dessen reine Nutzung, weswegen es sich also nicht um eine einfache Anwendung des bekannten Wortes „Kotvò tò $\varphi i ̂ \omega v^{4}$ handelt ${ }^{77}$. Man muß also berücksichtigen, daß der Terminus „Gemeinschaft" unterschiedliche (und weit flexiblere) Bedeutungsnuancen aufweist, die auch nicht unerheblich von dem abweichen, was wir heute unter „Vermögensgemeinschaft“ verstehen.

Was die Eigenart der verschmolzenen Vermögensmassen anbelangt, liefern die behandelten Autoren nur wenige Angaben; insbesondere ist nie von der Mitgift der Frau als einem wirtschaftlichen oder juristischen Phänomen die Rede, selbst wenn sie wohl

${ }^{76}$ Im übrigen riet offenbar auch Philodemos von Gadara dem epikureischen Weisen, die Reichtümer zu einer gemeinsamen Sache zu machen, und zwar in dem Sinne, daß er ihre Verwaltung mit vertrauten Freunden absprechen solle: vgl. Renato Laurenti, Filodemo (supra, A.13), 117122.

77 Vgl. supra, A.15. 
oft vorausgesetzt wird (zum Beispiel von Xenophon, Libanios und vielleicht auch Kallikratidas). Es ist durchaus denkbar, daß einige Texte, die der Frau das Recht einrāumen, die Güter des Mannes zu verwalten, implizit darauf abzielen, einen gewissen Parallelismus zwischen diesem Recht und demjenigen des Mannes an den Mitgiftgegenständen herzustellen. Doch sind die Aussagen der philosophischen Schriftsteller letzten Endes viel zu unbestimmt, als daß man derartige Úberlegungen anstellen dürfte; schließlich geht es in ihnen nicht um die Darstellung der Realităt, sondern um die Beschreibung eines Ideals.

Da stellt sich nun die Frage nach den Beziehungen zwischen den behandelten Theorien und der historischen Wirklichkeit: $Z$ war wird hier, wie gesagt, sicherlich nicht das durchschnittliche Familienleben dargestellt, heißt das aber, daß die Ausführungen als ganz wirklichkeitsfremd anzusehen sind oder daß sie keinerlei Einfluß auf die Praxis ausgeübt haben? Antworten auf solche Fragen sind schwer zu erteilen und können, selbst wenn der eine oder andere Hinweis im vorausgehenden schon gegeben worden sein mag, hier nicht einmal ansatzweise erteilt werden, würde dies doch Erörterungen erfordern, die den Umfang der bisherigen Ausführungen noch überstiegen. Statt dessen soll eine andere Frage gestellt werden, deren Beantwortung dem Leser überlassen sei: Könnten nicht vielleicht einige Ideale der antiken Philosophen auch noch in unserer heutigen Zeit Gültigkeit haben?

(Übersetzung: Carolina Cupane Kislinger) 
